\title{
CRESCIMENTO DE MUDAS DE CIPÓ-CRAVO (Tynanthus fasciculatus Miers), UMA LIANA COM POTENCIAL MEDICINAL ${ }^{1}$
}

Márdel M.M. Lopes², Rita Maria de Carvalho-Okano ${ }^{3}$, Agostinho Lopes de Souza ${ }^{4}$ e Haroldo Nogueira de Paiva $^{4}$

\begin{abstract}
RESUMO - O crescimento de mudas de cipó-cravo (Tynanthus fasciculatus Miers), liana com propriedades medicinais, foi avaliado em quatro condições: borda de mata (T1), interior de mata (T2), parte baixa de talude (T3) e parte alta de talude (T4), sendo plantadas 15 mudas em cada tratamento. O comprimento das plantas e o número de folhas foram registrados mensalmente por cerca de um ano. Para avaliar o crescimento em cada tratamento, foram ajustados modelos de regressão linear simples, comparados analisando-se a sobreposição nos intervalos de confiança obtidos. Houve diferença significativa entre T1, T2 e T3. A borda de mata foi o tratamento em que as mudas se desenvolveram melhor, ou seja, apresentaram 100\% de sobrevivência e os maiores comprimentos e número de folhas. Somente neste tratamento houve desenvolvimento de gavinhas em mudas que ultrapassaram $80 \mathrm{~cm}$ de comprimento.
\end{abstract}

Palavras-chave: Bignoniaceae, silvicultura e Mata Atlântica.

\section{SEEDLING GROWTH OF CLOVE VINE (Tynanthus fasciculatus Miers), A LIANA WITH MEDICINAL POTENTIAL}

\begin{abstract}
Seedling growth of clove-vine (Tynanthus fasciculatus Miers), a liana with medicinal properties, was evaluated under four conditions: forest edge (T1), forest interior (T2), bottom of sloping terrain (T3) and top of sloping terrain (T4). Fifteen seedlings were planted in each treatment. Plant length and number of leaves were recorded monthly for approximately one year. Growth in each treatment was adjusted to simple linear regression models, which were compared by overlapping the obtained confidence intervals. There were significant differences among T1, T2 and T3. Forest edge was the treatment in which seedlings grew better, showing $100 \%$ survival and the largest lengths and number of leaves; only in this treatment, the plants developed tendrils in seedlings over $80 \mathrm{~cm}$ long.
\end{abstract}

Keywords: Bignoniaceae, silviculture and Mata Atlantica.

\section{INTRODUÇÃO}

A família Bignoniaceae possui grande variedade de usos etnobotânicos e econômicos (SCHULTES e RAFFAUF, 1990; PHILLIPS, 1991; GENTRY, 1980, 1992, 1993). Além da importância na indústria madeireira e como planta ornamental, essa família detém grande potencial medicinal (GENTRY, 1992). De acordo com esse autor, há relato de 36 gêneros de Bignoniaceae que são utilizados na medicina popular: 27 gêneros de lianas e nove de árvores. Gentry (1993) citou que cerca de $38 \%$ das espécies de Bignoniaceae do noroeste da América do Sul se destina a algum uso específico, a maioria para fins medicinais.

\footnotetext{
${ }^{1}$ Recebido em 27.11.2006 e aceito para publicação em 20.02.2008.

${ }^{2}$ Herbário do Centro de Pesquisas do Cacau (CEPEC), Ilhéus-BA. E-mail : <mardelmml@ yahoo.com.br>.

${ }_{3}^{3}$ Departamento de Biologia Vegetal da Universidade Federal de Viçosa (UFV), Viçosa-MG. E-mail : <carvalho@ufv.br>.

${ }^{4}$ Departamento de Engenharia Florestal da UFV. E-mail : <alsouza@ufv.br>e <hnpaiva@ufv.br>.
} 
Espécies do gênero Tynanthus são lianas conhecidas popularmente como cipó-cravo, devido ao forte odor de cravo que exala dos caules, quando cortados ou macerados, e são usadas na medicina tradicional, para os mais diversos fins (GENTRY, 1992). Segundo Rizzini e Mors (1995), Tynanthus fasciculatus Miers é utilizada como afrodisíaco. Correa e Penna (1984) comentaram sobre a utilização de $T$. fasciculatus como planta ornamental, afirmando que seu caule exsuda água potável. Estudos farmacológicos sobre a ação anti-helmíntica da casca do caule dessa espécie, realizados por Amorim et al. (1991), mostraram redução significativa de verminoses em camundongos. Posteriormente, Amorim et al. (1994), com o intuito de avaliar o potencial toxicológico dessa planta, testaram a sua possível capacidade mutagênica em células de camundongo, o que não foi observado, levando-os à conclusão de que T. fasciculatus, como agente anti-helmíntico, possui índices terapêuticos bastante favoráveis.

T. fasciculatus apresenta distribuição relativamente restrita, ocorrendo no Sudeste brasileiro, em Minas Gerais, São Pauloe Rio de Janeiro (LOHMANN, no prelo; RIZZINI e MORS, 1995). A espécie apresenta folhas 3-folioladas, com o folíolo terminal geralmente modificado em gavinha trífida, flores pequenas, com a corola externamente branca e interiormente amarela, fruto seco, 4-alado e sementes aladas. Na região de Viçosa ( $20^{\circ} 45^{\prime}$ S, 4253'W), a espécie floresce de outubro a novembro, e os frutos amadurecem durante toda a estação seca e início da estação chuvosa; suas flores são visitadas, principalmente, por abelhas de médio porte, que são seus prováveis polinizadores (LOPES, 2005).

A exploração de plantas medicinais da flora nativa pelo extrativismo tem levado, muitas vezes, a reduções drásticas das populações naturais dessas plantas, seja pelo processo predatório de exploração, seja pelo desconhecimento dos seus mecanismos de perpetuação (REIS et al., 2000). Gentry (1993) comentou que, devido à exploração excessiva da casca de espécies de Tabebuia (Bignoniaceae), usada no tratamento de câncer e diabete, essas árvores estão localmente extintas ou muito raras em várias comunidades da Amazônia. O cipó-cravo, assim como a maioria dos produtos florestais nãomadeireiros da Mata Atlântica, é coletado sem plano de manejo, portanto sem preocupação com a sustentabilidade da espécie.

O manejo apropriado, fundamentado em conhecimentos tradicionais e, ou, em estudos da auto- ecologia das espécies de interesse farmacêutico, assim como a domesticação e o cultivo, são opções para obtenção de matéria-prima e redução do extrativismo desordenado nas florestas (REIS et al., 2000). Segundo esses autores, o cultivo de espécies nativas com propriedades medicinais deve ser estimulado; para as espécies pioneiras e secundárias iniciais, por se desenvolverem bem em ambientes mais ensolarados, esse cultivo ainda é mais fácil do que para espécies secundárias tardias.

Segundo Hartshorn (1995), a falta de informações ecológicas sobre espécies de liana é um dos principais impedimentos para o uso sustentável de produtos florestais não-madeireiros. De acordo com o autor, o fato de algumas espécies de liana possuírem propriedades farmacêuticas extraordinárias tem resultado em uma colheita descontrolada, e isso, aliado à ausência de cultivo, pode ameaçar a sobrevivência dessas espécies. As lianas são, geralmente, plantas heliófilas, e se desenvolvem melhor em ambientes com muita luminosidade, como clareiras e bordas (PUTZ, 1984; SANCHES e VÁLIO, 2002). Nos fragmentos de Florestas Estacionais Semideciduais, em Viçosa, Minas Gerais, muitas espécies de Bignoniaceae se distribuem abundantemente ao longo das bordas, inclusive $T$. fasciculatus.

Considerando o potencial de utilização de $T$. fasciculatus como produto florestal não-madeireiro, e sua possibilidade de uso na recomposição de ambientes, este trabalho teve como objetivo avaliar o crescimento de mudas dessa espécie em diferentes tratamentos.

\section{MATERIAL E MÉTODOS}

Em outubro de 2003, 615 sementes foram plantadas em sacolas plásticas de $15 \mathrm{~cm}$ de altura por $7 \mathrm{~cm}$ de diâmetro, contendo substrato argiloso, enriquecido com fósforo, na forma de superfosfato simples, na proporção de $5 \mathrm{~kg} / \mathrm{m}^{3}$. Três sementes foram colocadas na superfície do substrato de cada sacola e cobertas com serragem de eucalipto, para evitar que se dispersem com o vento. As sacolas foram colocadas em um canteiro ao ar livre, irrigado duas vezes ao dia, quando não chovia. Nas sacolas em que germinaram mais de uma semente, foi feita a repicagem das plântulas.

Após 110 dias da semeadura, realizou-se a adubação de cobertura, com solução nutritiva de nitrogênio, na forma de sulfato de amônio (10 g/L) e potássio, na 
forma de cloreto de potássio (3 g/L), colocando-se $20 \mathrm{~mL}$ por sacola. Para o plantio no campo, foram selecionadas as 60 melhores mudas, que tinham em média $10 \mathrm{~cm}$ de altura, variando entre 7 e $15 \mathrm{~cm}$.

As mudas foram plantadas em março de 2004, em quatro tratamentos: T1 - borda de mata; T2 - interior de mata; T3 - parte baixa de talude; e T4 - parte alta de talude. Os dois primeiros tratamentos foram instalados na Estação de Pesquisa, Treinamento e Educação Ambiental Mata do Paraíso (EPTEAMP), fragmento de mata secundária, preservado desde 1965 (MARANGON, 2003), classificado como Floresta Estacional Semidecidual Montana (VELOSO, 1991), com aproximadamente 194 ha. AEPTEAMP situa-se em Viçosa, Minas Gerais, sendo o maior fragmento florestal da região (RIBON, 2005). Os dois últimos tratamentos foram instalados em um talude localizado no sítio Ipê, propriedade rural situada a uma distância de aproximadamente $2 \mathrm{~km}$ daEPTEAMP. Em cada tratamento foram plantadas 15 mudas. As covas mediam cerca de $20 \times 20 \times 20 \mathrm{~cm}$, sendo adicionados $100 \mathrm{~g}$ de superfosfato simples por cova, nos quatro tratamentos. O comprimento (da base ao ápice do caule) de cada indivíduo foi medido, mensalmente, de abril de 2004 a março de 2005 e em junho de 2005. De cada tratamento, foram sorteados cinco indivíduos que, a partir de maio de 2004, além do comprimento, tiveram o número de folhas registrado.

Foram ajustados modelos de regressão linear simples para avaliar o crescimento em cada tratamento, que foram comparados, verificando-se a sobreposição entre os intervalos de confiança.

\section{RESULTADOS E DISCUSSÃO}

Não houve sobreposição entre os intervalos de confiança dos tratamentos T1, T2 e T3, evidenciandose que houve diferença significativa entre estes (Quadro 1). Os maiores valores do comprimento foram observados na borda de mata (T1), seguidos pelos tratamentos do interior de mata (T2) e da parte baixa de talude (T3; Figura 1). Não foi possível fazer a análise de regressão linear do tratamento da parte alta de talude (T4), por causa da ocorrência mais intensa de herbivoria e seca de ápices caulinares, o que gerou decréscimo no comprimento de diversas plantas ao longo do período de estudo (Figura 1).

Na borda de mata (T1) foram encontrados os maiores comprimentos no final do experimento (Figura 1). Esse resultado corrobora os dados de um experimento realizado em Floresta Estacional Semidecidual em Campinas, SP (SANCHES e VÁLIO, 2002). Em floresta tropical no Equador, Nabe-Nielsen (2002) mostrou que, quanto maior a abertura do dossel, maior o crescimento das plântulas de Machaerium cuspidatum Kuhlm. \& Hoehne (Leguminosae). O mesmo autor citou experimento realizado por Baars e Kelly (1996), no qual espécies de lianas cresciam mais rapidamente quando expostas a níveis elevados de luz.

Na mata, o número médio de folhas manteve-se relativamente constante, tanto no interior quanto na borda, porém sempre maior nesta (Figura 2), semelhantemente ao observado por Sanches e Válio (2002) em 17 espécies de trepadeiras. No talude, as mudas apresentaram uma queda drástica no número médio de folhas em maio/junho, estação seca em Viçosa, voltando a emitir folhas em outubro (Figura 2), início da estação chuvosa. Na última medição, os cinco indivíduos avaliados na parte alta do talude estavam completamente desfolhados.

Das 60 mudas plantadas, 12 morreram: quatro no interior de mata; seis na parte baixa de talude; e duas na parte alta de talude. Na borda da mata, todas as mudas sobreviveram. Em janeiro e fevereiro de 2005, houve ataque leve de insetos herbívoros na borda e no interior da mata. No talude, a herbivoria, provavelmente por formigas-cortadeiras (Atta sp.), foi mais intensa, ocorrendo, sobretudo, em junho de 2004.

Quadro 1 - Valores de probabilidade (P), coeficiente de determinação $\left(R^{2}\right)$, inclinação (b1) e os limites inferiores e superiores dos intervalos de confiança

Table 1 - Probability $(P)$, coefficient of determination $\left(R^{2}\right)$, slope (b1) values and inferior and superior limits of confidence intervals

\begin{tabular}{lcccc}
\hline Tratamento & $\mathrm{P}$ & $\mathrm{R} 2$ & $\mathrm{~b} 1$ & \multicolumn{2}{c}{ Intervalo de Confiança (95\%) } \\
\cline { 4 - 5 } & & & & Limite Inferior \\
\hline Borda de mata & 0,0000154 & 0,95 & 0,1232 & 0,1046 \\
Interior de mata & 0,000000559 & 0,90 & 0,0195 & 0,0153 \\
Parte baixa de talude & 0,0000215 & 0,80 & 0,0079 & 0,0054 \\
\hline
\end{tabular}

R. Árvore, Viçosa-MG, v.32, n.2, p.211-216, 2008 
$\mathrm{O} \mathrm{IM} \times \mathrm{BM} \triangle \mathrm{TA} \square \mathrm{TB}$

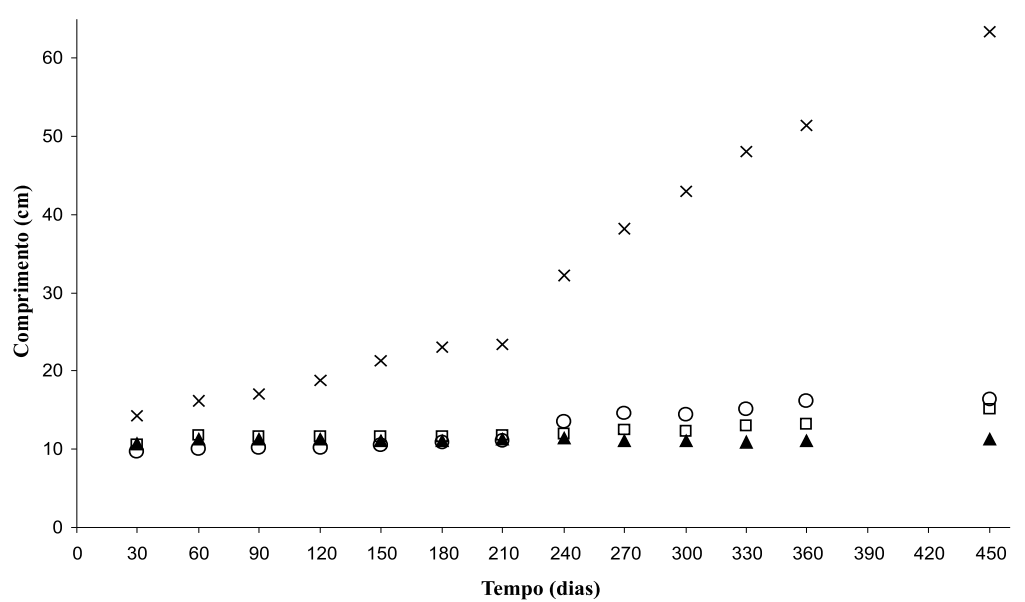

Figura 1 - Comprimento das plantas em função do tempo, no interior de mata (IM), borda de mata (BM), parte alta de talude (TA) e parte baixa de talude (TB), de abril/2004 a junho/2005.

Figure 1 - Plant length in function of time, in the forest interior (IM), forest edge (BM), bottom of sloping terrain (TB) and top of sloping terrain (TA), from April/2004 to June/2005.

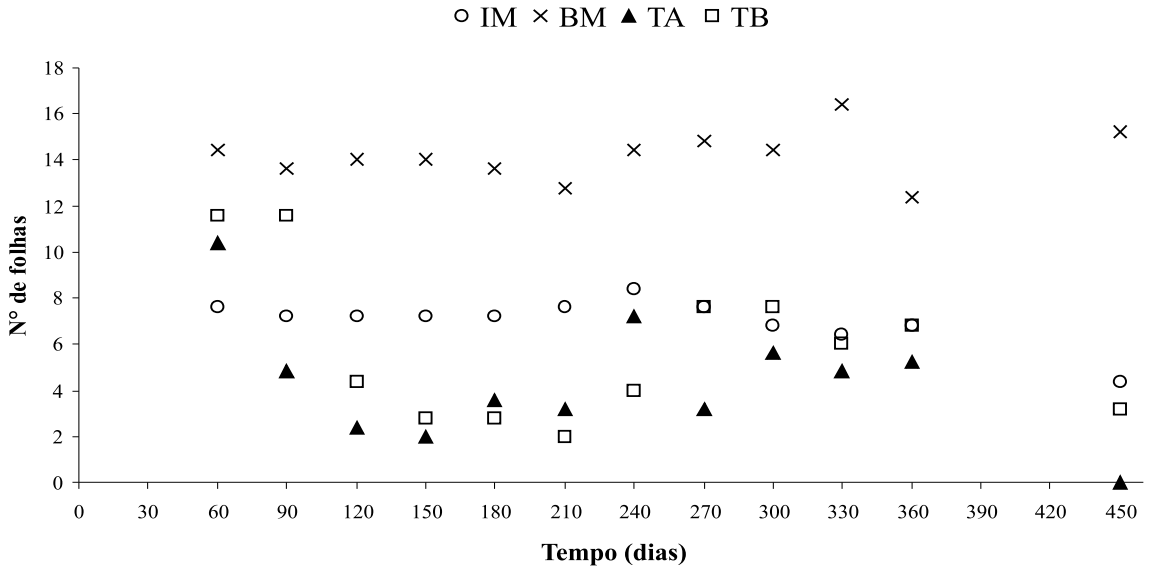

Figura 2 - Número médio de folhas de T. fasciculatus em função do tempo, no interior de mata (IM), borda de mata (BM), parte alta de talude (TA) e parte baixa de talude (TB), de maio/2004 a junho/2005.

Figure 2 - Average number of leaves of T. fasciculatus, in function of time, in the forest interior (IM), forest edge (BM), sloping terrain bottom (TB) and sloping terrain top (TA), from May/2004 to June/2005.

Gavinhas desenvolveram-se apenas nas quatro maiores mudas da borda da mata, quando estas ultrapassaram $80 \mathrm{~cm}$ de comprimento, indicando que o mecanismo envolvido na diferenciação das gavinhas está mais relacionado com o comprimento do que com a idade da planta.

O plantio de espécies com potencial econômico ou utilização etnobotânica deve ser incentivado, na tentativa de conservar as populações dessas espécies e promover alternativas econômicas que incentivem a manutenção e recomposição de fragmentos florestais. T. fasciculatus desenvolveu-se notadamente melhor na borda do que no interior da mata e no talude. Dessa forma, na região de Viçosa as bordas de fragmentos florestais ou, mesmo, de sistemas agroflorestais seriam os ambientes mais propícios ao crescimento dessa espécie, pensando-se em futuros plantios. 


\section{AGRADECIMENTOS}

Ao Departamento de Engenharia Florestal, pelo espaço concedido no viveiro e na EPTEAMP; e aos seus funcionários Geraldo Magela, pelo auxílio nos trabalhos de viveiro, e Márcio e Machado, pela coleta das sementes; à Helga Arato, pela ajuda na coleta de dados; ao Marcio Oliveira, pelo grande auxílio nos cálculos estatísticos; ao Roberto Bloomfield, por ceder o terreno para a avaliação do crescimento em talude; e à CAPES, pela bolsa de mestrado.

\section{REFERÊNCIAS}

AMORIM, A.; BORBA, H. R.; AMANO, L. M. Ação anti-helmíntica de plantas IV: influência da casca do caule de cipó-cravo (Tynanthus fasciculatus Miers; Bignoniaceae) na eliminação de Vampirolepis nana e de oxiurídeos em camundongos. Revista Brasileira de Farmácia, v.72, n.4, p.94-92, 1991.

AMORIM, A. et al. Testes de mutagenicidade em camundongos tratados com extratos aquosos de Tynanthus fasciculatus Miers (cipó-cravo). Revista Brasileira de Farmácia, v.75, n.2, p.46-47, 1994.

CORREA, M. P.; PENNA, L. A. Dicionário das plantas úteis do Brasil e das exóticas cultivadas. Rio de Janeiro: Imprensa Nacional, 1984. v.2. 638p.

GENTRY, A. H. Bignoniaceae. Part I (Crescentieae and Tourrettieae). New York: The New York Botanical Garden, 1980. 130p. (Flora Neotropica, v.25)

GENTRY, A. H. A synopsis of Bignoniaceae ethnobotany and economic botany. Annals of the Missouri Botanical Garden, v.79, n.1, p.53-64, 1992.

GENTRY, A. H. Tropical forest biodiversity and the potential for new medicinal plants. In:

KINGHORN, A. D.; BALANDRIN, M. F. (Eds.).

Human medicinal agents from

plants. Washington: American Chemical

Society, 1993. p.13-24.

HARTSHORN, G. S. Ecological basis for sustainable development in tropical forests.

Annual Review of Ecology and Systematics, v.26, p.155-175, 1995.
LOHMANN, L. G. A new generic classification of Bignonieae (Bignoniaceae) based on molecular phylogenetic data and morphological synapomorphies. Annals of Missouri Botanical Garden, no prelo.

LOPES, M. M. M. Bignoniaceae Durande de um fragmento florestal, em Viçosa, zona da mata mineira: florística e aspectos ecológicos. 2005. 111f. Dissertação (Mestrado em Botânica) - Universidade Federal de Viçosa, Viçosa, MG, 2005.

MARANGON, L. C.; SOARES, J. J.; FELICIANO, A. L. P. Florística arbórea da mata da pedreira, município de Viçosa, Minas Gerais. Revista Árvore, v.27, n.2, p.207-215, 2003.

NABE-NIELSEN, J. Growth and mortality rates of the liana Machaerium cuspidatum in relation to light and topographic position. Biotropica, v.34, n.2, p.319-322, 2002.

PHILLIPS, O. The ethnobotany and economic botany of tropical vines. In: PUTZ, F. E.; MOONEY, H. A (Eds.). The biology of vines. Cambridge: Cambridge University Press, 1991. p.427-475.

PUTZ, F. E. The natural history of lianas on Barro Colorado Island, Panama. Ecology, v.65, n.6, p.1713-1724, 1984.

REIS, M. S.; MARIOT, A.; DI STASI, L. C. Manejo de populações naturais de plantas medicinais na Floresta Atlântica. In: DIEGUES, A. C.; VIANA, V. M. (Orgs.). Comunidades tradicionais e manejo dos recursos naturais da Mata Atlântica. São Paulo: NUPAUB-USP, 2000. p.95-102.

RIBON, R. Demarcação de uma grade de trilhas no centro de pesquisas da Mata do Paraíso, Viçosa, Minas Gerais. Revista Árvore, v.29, n. 1, p.151-158, 2005.

RIZZINI, C. T.; MORS, W. R. Botânica econômica brasileira. 2.ed. Rio de Janeiro: Âmbito Cultural, 1995. 248p.

SANCHES, M. C.; VÁLIO, I. F. M. Seedling growth of climbing species from a southeast brazilian tropical forest. Plant Ecology, v.159, n.1, p.51-59, 2002.

R. Árvore, Viçosa-MG, v.32, n.2, p.211-216, 2008 
SCHULTES, R. E.; RAFFAUF, R. F. The

healing forest: Medicinal and toxic plants of the northwest Amazonia. Portland: Dioscorides Press, 1990. 484p.
VELOSO, H. P.; FILHO, A. L. R. R. \& LIMA, J. C. A. Classificação da vegetação brasileira, adaptada a um sistema universal. Rio de Janeiro: IBGE, 1991. 123p. 